\title{
Comparison Between Two Methods for Delayed Decision Feedback Sequence Estimation
}

\author{
Maurizio Magarini, Arnaldo Spalvieri, and Guido Tartara, \\ Dipartimento di Elettronica e Informazione, Politecnico di Milano, \\ Piazza Leonardo da Vinci, 32, I-20133 Milano (Italy). \\ e-mail: magarini@elet.polimi.it
}

\begin{abstract}
The paper deals with the design of suboptimal receivers for data transmission over frequency selective channels. The complexity of Maximum Likelihood Sequence Estimation (MLSE) turns out be exponential in the channel memory. Hence, when dealing with channels with long memory, suboptimal reception must be considered. Among suboptimal methods, the Delayed Decision Feedback Sequence Estimation (DDFSE) plays an important role. This receiver is based on a Viterbi processor where the channel memory is truncated. The memory truncation is compensated by a per-survivor decision feedback equalizer. The DDFSE was originally proposed in [1], where the whitened matched filter was considered as a front-end. Our contribution is to extend the principles of MLSE and DDFSE to the case where the mean-square whitened matched filter is adopted as a front-end. Simulation results show that our proposed design of the DDFSE gives substantial benefits when a severe frequency selective channel is considered.
\end{abstract}

\section{INTRODUCTION}

The increasing demand for wideband communication services has lead to the development of broadband transmission systems. Both in the fixed and in the mobile scenario, radio systems are subject to propagation over multipath channels. The effect of multipath is severe in broadband systems, where the symbol repetition interval may be shorter than the delay between the paths. In this case, one or more deep notches affect the spectrum of the received signal. Equalization attempts to recover the transmitted data from the received waveform by suitable processing of the received signal. The receiver that guarantees minimum Bit Error Rate (BER) is the maximum a posteriori probability receiver. However, one often renounces to this receiver, because it is too complex. A simpler receiver is obtained if the probability of sequence error is considered. This approach leads to MLSE $[2,3,4]$. Unfortunately, even MLSE is often too complex. Actually, the MLSE receiver is realized by a Viterbi algorithm with a number of states that is exponential in the channel memory. Hence, when dealing with channels with long memory, one is forced to consider suboptimal receivers. A popular one is based on the Decision Feedback Equalizer (DFE) [5]. The complexity of the DFE is much lower than the complexity of MLSE. Unfortunately, the gap between the performances of the two detectors is huge when a severe frequency selective channel is considered. A technique that allows for a trade off between complexity and performance is the DDFSE proposed in [1]. The DDFSE is based on a Viterbi algorithm where the channel memory is truncated. The performance loss due to memory truncation is mitigated by a per-survivor processing [6], where the past history of each survivor is used in a DFE scheme. In the DDFSE originally proposed in [1], the Whitened Matched Filter (WMF) was considered as a front-end. Our contribution is to extend MLSE and DDFSE to the case where the Mean-Square Whitened Matched Filter (MSWMF) is adopted as a front-end. The outline of the paper is as follows. In Section 2 MLSE based on the WMF and on the MSWMF is reported, and in the Appendix it is proved that the MSWMF leads to MLSE with minimum number of states. Section 3 describes the WMF-DDFSE and the MSWMF-DDFSE. Simulation results, reported in section 4 , show that the MSWMF-DDFSE outperforms the WMF-DDFSE at low-to-intermediate SNR when a severe frequency selective channel is considered. In section 5 the summary of the results is presented.

\section{TWO MLSE METHODS}

We consider the model of a binary uncoded data sequence transmitted over a baseband linear channel corrupted by additive white Gaussian noise. It is well known that MLSE can be performed by a sampled matched filter, a prefilter, and Viterbi algorithm, 
Figure 1: Channel and receiver block diagram.

and that the branch metric to be used in the algorithm can be calculated in several ways, depending on the prefilter. Examples are the receivers described in $[2,3,4]$. We consider two receivers that allow for MLSE with minimum number of states making use of the familiar squared Euclidean distance as a branch metric. The block diagram of the system is reported in figure 1. Let $2^{\nu}$ be the number of states and $2^{\nu+1}$ the number of transitions. The metric of the transition that diverges from state $\left(a_{k-\nu}, \ldots, a_{k-1}\right)$ at time $k-1$ and merges in state $\left(a_{k-\nu+1}, \ldots, a_{k}\right)$ at time $k$ is

$$
b_{k}\left(a_{k-\nu}, \ldots, a_{k}\right)=\left(x_{k}-\sum_{j=0}^{\nu} d_{j} a_{k-j}\right)^{2},
$$

where $x_{k}$ is the $k$ th sample at the output of the prefilter, and $a_{k} \in\{-1,1\}$. The impulse response $\mathbf{d}=\left(d_{0}, d_{1}, \ldots, d_{\nu}\right)$ and the prefilter are hereafter discussed for the two receivers.

\section{A. Whitened Matched Filter}

Let $\mathbf{r}=\left(r_{-\nu}, \ldots, r_{\nu}\right)$ be the impulse response of the system from the source to the output of the sampled matched filter, that is the sampled autocorrelation of the channel $g(t)$ in figure 1 , and let $r(z)=\sum_{i=-\nu}^{\nu} r_{i} z^{-i}$ be its $z$-transform $\left(z^{-1}\right.$ represents the unit delay). In the time discrete white Gaussian model of [2] $d(z)$ is obtained from the spectral factorization

$$
d(z) d\left(z^{-1}\right)=r(z)
$$

by taking for $d_{W M}(z)$ that $d(z)$ being causal and minimum phase. It is worth noting that $d_{W M}(z)$, that is $d(z)$ causal and minimum phase, exists iff the Fourier transform

$$
S(\omega)=r\left(e^{j \omega}\right),
$$

is nonnull over any measurable interval [7]. When this condition holds true, the roots of $d_{W M}(z)$ are on or inside the unit circle. Note that the case where some of the roots of $d_{W M}(z)$ are on the unit circle, that is when $S(\omega)$ is null in some non measurable interval, is a limiting case. When the roots of $d_{W M}(z)$ are inside the unit circle, the prefilter is the noise whitening filter

$$
e_{W M}(z)=d_{W M}^{-1}\left(z^{-1}\right)
$$

When the roots of $d_{W M}(z)$ are on the unit circle, the noise whitening filter does not exist, because
$d_{W M}\left(z^{-1}\right)$ is not invertible. However, the existence of the WMF is still guaranteed [2], and the impulse response of the system from the source to the input to the Viterbi detector is $d(z)$.

\section{B. Mean Square Whitened Matched Filter}

In the second receiver that we consider, $d(z)$ is determined from the spectral factorization

$$
d(z) d\left(z^{-1}\right)=r(z)+\sigma^{2}
$$

by taking for $d_{M S}(z)$ that $d(z)$ being causal and minimum phase. In (5), $\sigma^{2}$ is the variance of the noise at the output of the sampler. Note that for $\sigma>0$, that is when the detection problem is not trivial, the Fourier transform $S(\omega)+\sigma^{2}$ is nonnull everywhere. As a consequence, the existence of $d_{M S}(z)$ is guaranteed, all its roots are strictly inside the unit circle, and, in contrast to the WMF, the case where $S(\omega)$ is null over some interval is not a limiting case for the MSWMF. Also note that the time spanning of $r(z)+\sigma^{2}$ is the same as that of $r(z)$, hence $d_{M S}(z)$ has the same time spanning as $d_{W M}(z)$, leading to a Viterbi algorithm with the same number of states as for the WMF, that is the minimum number of states for MLSE [4]. The prefilter is the filter that minimizes the Mean Square Error (MSE), where the MSE is defined as

$$
M S E=E\left\{\left(x_{k}-\sum_{i=0}^{\nu} d_{M S, i} \tilde{a}_{k-i}\right)^{2}\right\},
$$

where $E\{\cdot\}$ denotes the expected value, and $\tilde{a}_{k}$ is the $k$ th element of the transmitted sequence. Minimization of (6) yields [7]

$$
e_{M S}(z)=\frac{d_{M S}(z)}{r(z)+\sigma^{2}}=d_{M S}^{-1}\left(z^{-1}\right)
$$

provided that $\sigma>0$. It can be shown that the error sequence $\left\{x_{k}-\sum_{i=0}^{\nu} d_{M S, i} \tilde{a}_{k-i}\right\}$ is white [7]. For this reason, the front-end filter takes the name of mean-square whitened matched filter. Although (5) is widely known and used in the theory and in the practice of DFE, we are unaware of its use in MLSE. Hence we state the following

Theorem : A Viterbi detector based on (1), (5), and (7), performs MLSE.

The theorem is proved in the appendix. 

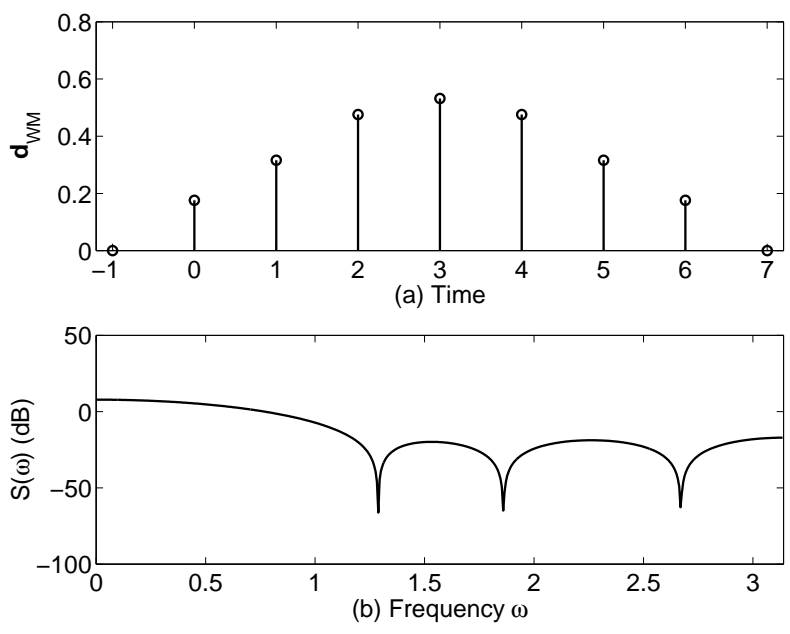

Figure 2: (a) Discrete time channel. (b) Spectrum.

\section{TWO DDFSE METHODS}

In the DDFSE, $d(z)$ is split in two parts as

$$
\begin{aligned}
d_{1}(z) & =\sum_{i=0}^{\mu} d_{i} z^{-i}, \\
d_{2}(z) & =\sum_{i=\mu+1}^{\nu} d_{i} z^{-i}, \\
d(z) & =d_{1}(z)+d_{2}(z) .
\end{aligned}
$$

The Viterbi algorithm with $2^{\mu}$ states, $\mu \leq \nu$, treats in a conventional manner $d_{1}(z)$, while the $\nu-\mu$ taps of the per-survivor DFE are used to cancel the intersymbol interference caused by $d_{2}(z)$. The branch metric in the reduced trellis is

$$
\begin{aligned}
b_{k}\left(a_{k-\mu}, \ldots, a_{k}\right)=\left(x_{k}-\sum_{j=0}^{\mu} d_{j} a_{k-j}\right. \\
\left.-\sum_{j=\mu+1}^{\nu} d_{j} \hat{a}_{k-j}\left(a_{k-\mu}, \ldots, a_{k-1}\right)\right)^{2},
\end{aligned}
$$

where $\hat{a}_{k-j}\left(a_{k-\mu}, \ldots, a_{k-1}\right)$ is the estimate of the bit transmitted at time $k-j$ which is present in the survivor that at time $k-1$ merges in the state $\left(a_{k-\mu}, \ldots, a_{k-1}\right)$. As mentioned in the introduction, in the DDFSE introduced in [1] the WMF is considered as a front-end. Note that, when $\mu=0$, the DDFSE reduces to a pure DFE. We observe that the spectral factorization (5), that is called the key equation in [7], plays a central role in the theory of the pure DFE, while (2) is less important. This observation suggests to adopt the MSWMF as a front-end also for DDFSE.

\section{EXPERIMENTAL RESULTS}

To obtain substantial difference between the performances of the two methods, a severely distorted
Figure 3: Trade off between complexity and performance for the two competitors. SNR=20 dB. $\mu=0$ is the pure DFE, $\mu=6$ is MLSE.

channel should be considered. The channels studied in [8] are actually severe, in the sense that they give the lower minimum distance for a fixed length of the impulse response. We focus on the channel with $\nu=6$. The impulse response of the time discrete channel at the output of the WMF, depicted in figure 2 together with the spectrum $S(\omega)$, is $d_{W M}(z)=0.176+0.316 z^{-1}+0.476 z^{-2}+0.532 z^{-3}+$ $0.476 z^{-4}+0.316 z^{-5}+0.176 z^{-6}$. The shape of the impulse response resembles a bell, a shape that is often found in channels from the real world. Note that this channel has three spectral nulls. It is intuitive that the effect of the spectral nulls is more severe for the WMF-DDFSE, where the spectral nulls are treated as a limiting case, than for the MSWMFDDFSE. Moreover, it is apparent from (2) and (5) that $d_{M S}(z) \rightarrow d_{W M}(z)$ as SNR $\rightarrow \infty$. Hence one expects that the MSWMF-DDFSE outperforms his competitor only at low-to-intermediate SNR. However, the important issue of performance evaluation is left to future investigation, limiting the present contribution to simulation results. In the simulations, the sequence at the output of the WMF is $x_{W M}(z)=$ $d_{W M}(z) \tilde{a}(z)+w(z)$, where the variance of the white noise $w(z)$ is $\sigma^{2}$. For the MSWMF, $r(z)$ is calculated from (2), then is used in (5) to work out $d_{M S}(z)$. The sequence at the output of the MSWMF is then obtained as $x_{M S}(z)=x_{W M}(z) e_{W M}^{-1}(z) e_{M S}(z)=$ $x_{W M}(z) d_{W M}\left(z^{-1}\right) d_{M S}^{-1}\left(z^{-1}\right)$, the existence of $d_{M S}^{-1}\left(z^{-1}\right)$ being guaranteed for $\sigma>0$. The product $d_{W M}\left(z^{-1}\right) d_{M S}^{-1}\left(z^{-1}\right)$ is truncated to 91 terms. The $\mathrm{BER}$ is measured by a random sequence of $2 \cdot 10^{6}$ data. Figure 3 reports the BER versus $\mu$ at SNR $=r_{0} / \sigma^{2}=20 \mathrm{~dB}$. The figure illustrates the trade off between performance and complexity offered by the DDFSE, and shows that the MSWMF-DDFSE outperforms the WMF-DDFSE. Of course, for $\mu=6$ both receivers perform MLSE. Figure 4 shows the 
BER of the pure DFE $(\mu=0)$ versus SNR, confirming the observation that leaded us to consider the MSWMF as a front-end. Figure 5 , where $\mu=4$ is considered, demonstrates the improvement offered by the MSWMF-DDFSE over his competitor in a realistic trade off between complexity and performance. Figures 4 and 5 also show that, as expected, the improvement offered by the MSWMF-DDFSE over his competitor is higher at low-to-intermediate SNR.

\section{SUMMARY}

The MSWMF is widely known and studied in the theory of DFE, but seems to be less considered in sequence estimation. Moving from this observation, we have proved that MLSE with minimum number of states is obtained when the MSWMF is adopted as a front-end. Further, the MSWMF has been proposed as a front-end for the suboptimal sequence estimation technique known as DDFSE. The comparison between the MSWMF-DDFSE and the conventional WMF-DDFSE shows that the MSWMF-DDFSE outperforms his rival when a severe frequency selective channel is considered. This result is intuitive, because the receiver based on the WMF treats spectral nulls as a limiting case. In contrast, the case where the spectrum is null in some interval is not a limiting case for the MSWMF, provided that $\mathrm{SNR} \neq \infty$. Simulation results also confirm that, since MSWMF $\rightarrow \mathrm{WMF}$ as $\mathrm{SNR} \rightarrow \infty$, a substantial improvement is offered by the MSWMF over the WMF only at lowto-intermediate SNR. In the present paper, the performance is evaluated by computer simulation, while the analysis is left to further study.

\section{APPENDIX}

\section{Theorem :}

A Viterbi detector based on (1), (5), and (7), performs MLSE.

\section{Proof :}

Let $\mathbf{s}$ be the survivor that merges in state $\mathbf{s}_{k}=$ $\left(a_{k-\nu+1}, \ldots, a_{k}\right)$ at time $k$, let $\mathbf{a}=\left(a_{k+1}, \ldots, a_{k+l}\right)$ be a piece of a generic data sequence that diverges from state $\mathbf{s}_{k}$ at time $k$ and merges at time $k+l$, $l \geq \nu+1$, in state $\mathbf{s}_{k+l}=\left(a_{k-\nu+1+l}, \ldots, a_{k+l}\right)$, and let $\mathbf{y}=\left(y_{k+1}, \ldots, y_{k+l}\right)$ be the corresponding piece of sequence of the samples at the output of the sampled matched filter. Manipulating the branch metric of Ungerboeck' s MLSE [3], one writes the metric of $(\mathbf{s}, \mathbf{a})$ at time $k+l$ as

$$
\begin{aligned}
M_{k+l}^{M F}(\mathbf{s}, \mathbf{a})= & \left(\mathbf{s}_{k}, \mathbf{a}\right) \mathbf{R}_{l+\nu}\left(\mathbf{s}_{k}, \mathbf{a}\right)^{T} \\
& -2 \mathbf{y} \mathbf{a}^{T}+M_{k}^{M F}(\mathbf{s}, \mathbf{a})-\mathbf{s}_{k} \mathbf{R}_{\nu} \mathbf{s}_{k}^{T}
\end{aligned}
$$

where $M_{k}^{M F}(\mathbf{s}, \mathbf{a})$ is the metric of state $\mathbf{s}_{k}$ at time $k$, $\mathbf{R}_{m}$ is the conventional $m \times m$ autocorrelation ma-

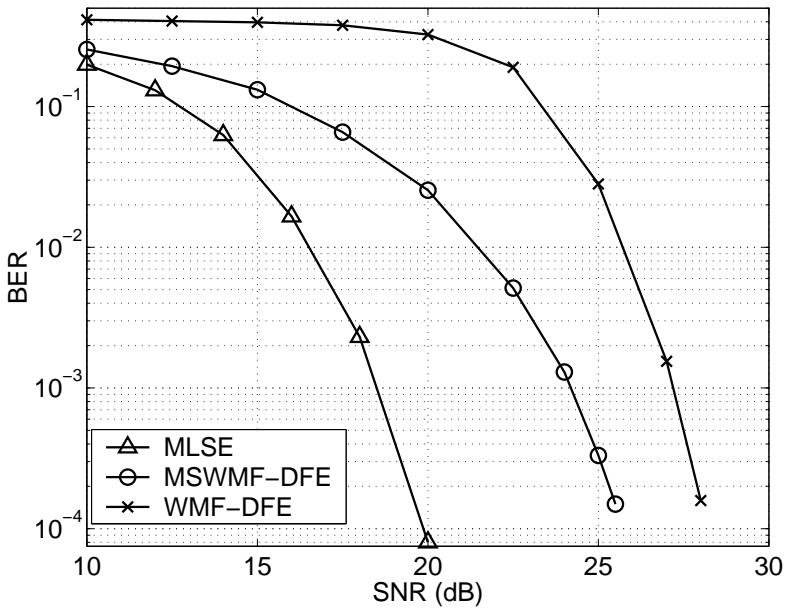

Figure 4: BER versus SNR for the pure DFE.

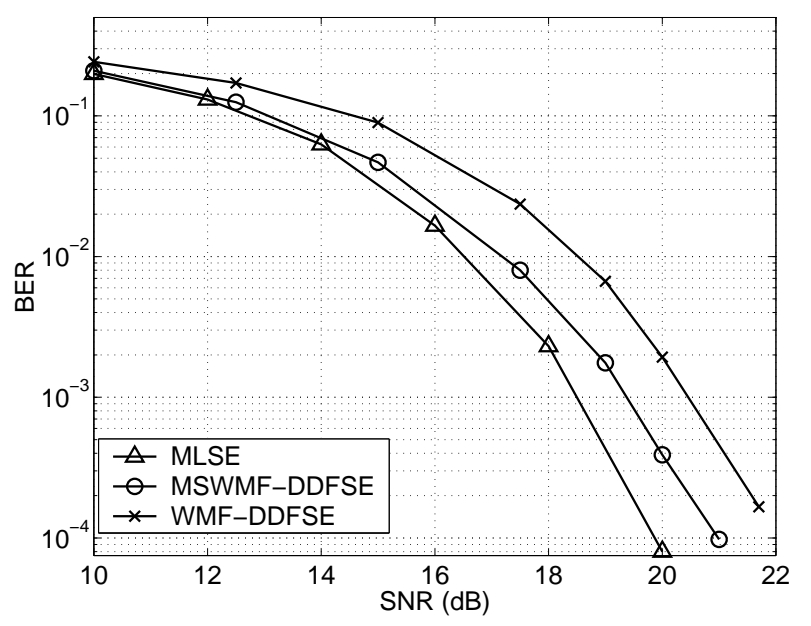

Figure 5: BER versus SNR for reduction of the Viterbi algorithm to 16 states.

trix constructed from $\mathbf{r}$, and the superscript ${ }^{T}$ denotes transposition. Note that the term $M_{k}^{M F}(\mathbf{s}, \mathbf{a})-$ $\mathbf{s}_{k} \mathbf{R}_{\nu} \mathbf{s}_{k}^{T}$ is common to all the sequences that diverge from $\mathbf{s}_{k}$. Consider now the decision between two sequences $\left(\mathbf{s}, \mathbf{a}^{\prime}\right)$ and $\left(\mathbf{s}, \mathbf{a}^{\prime \prime}\right)$ that diverge from state $\mathbf{s}_{k}$ at time $k$ and merge at time $k+l$ in state $\mathbf{s}_{k+l}$. The test takes the form

$$
M_{k+l}^{M F}\left(\mathbf{s}, \mathbf{a}^{\prime}\right) \gtrless M_{k+l}^{M F}\left(\mathbf{s}, \mathbf{a}^{\prime \prime}\right) .
$$

The same decision rule is obtained by considering the following two iterations. The first iteration is

$$
\begin{aligned}
u_{k}=0, \quad u_{j+1}=u_{j}+\left(\sum_{i=0}^{\nu} d_{M S, i} a_{j-i+1}\right)^{2}, \\
j=k, \ldots, k+l-1 .
\end{aligned}
$$

Manipulating (9) one finds

$$
\begin{aligned}
& u_{k+l}=\left(\mathbf{s}_{k}, \mathbf{a}\right)\left(\mathbf{R}_{l+\nu}+\sigma^{2} \mathbf{I}_{l+\nu}\right)\left(\mathbf{s}_{k}, \mathbf{a}\right)^{T} \\
& -\sum_{n=k+1}^{k+\nu}\left(\sum_{i=k-\nu+1}^{k} d_{M S, n-\nu-i} a_{i}\right)^{2}
\end{aligned}
$$




$$
-\sum_{n=k+l+1}^{k+l+\nu}\left(\sum_{i=k+l-\nu+1}^{k+l} d_{M S, n-i} a_{i}\right)^{2}
$$

where $\mathbf{I}_{m}$ is the $m \times m$ identity matrix, and the two double sum and the term $\left(\mathbf{s}_{k}, \mathbf{a}\right) \sigma^{2} \mathbf{I}_{l+\nu}\left(\mathbf{s}_{k}, \mathbf{a}\right)^{T}$ are common to all the sequences that diverge from and merge in the two states mentioned above (recall binary antipodal transmission and $d_{M S, i}=0$ for $i \notin\{0, \ldots, \nu\})$. The second iteration is based on

$$
\begin{aligned}
\mathbf{y a}^{T} & =\left[y(z) a\left(z^{-1}\right)\right]_{0} \\
& =\left[y(z) e_{M S}(z) d_{M S}\left(z^{-1}\right) a\left(z^{-1}\right)\right]_{0} \\
& =\left[x(z) d_{M S}\left(z^{-1}\right) a\left(z^{-1}\right)\right]_{0},
\end{aligned}
$$

where $[\cdot]_{i}$ denotes the coefficient of $z^{i}$, and $e_{M S}(z) d_{M S}\left(z^{-1}\right)=1$ from (7) has been used. From (10) one gets

$$
\begin{aligned}
& v_{k}=0, v_{j+1}=v_{j}+x_{j+1} \sum_{i=0}^{\nu} d_{M S, i} a_{j-i+1}, \\
& j=k, \ldots, k+l-1,
\end{aligned}
$$

whose last term, after some manipulation, can be written as

$$
\begin{aligned}
v_{k+l}= & \mathbf{y a}^{T}+\sum_{n=k+1}^{k+\nu} x_{n}\left(\sum_{i=k-\nu+1}^{k} d_{M S, n-i} a_{i}\right) \\
& -\sum_{n=k+l+1}^{k+l+\nu} x_{n}\left(\sum_{i=k+l-\nu+1}^{k+l} d_{M S, n-i} a_{i}\right),
\end{aligned}
$$

where the two double sum are common to all the sequences that diverge from and merge in the two mentioned states. Putting together (9), (11), and adding $x_{j+1}^{2}$, which is common to all the sequences, one gets

$$
\begin{aligned}
& M_{j+1}^{M S}(\mathbf{s}, \mathbf{a})=M_{j}^{M S}(\mathbf{s}, \mathbf{a})+\left(x_{j+1}\right. \\
& \left.-\sum_{i=0}^{\nu} d_{M S, i} a_{j-i+1}\right)^{2}, \quad j=k, \ldots, k+l-1 .
\end{aligned}
$$

Disregarding all the common terms, one realizes that the test

$$
M_{k+l}^{M S}\left(\mathbf{s}, \mathbf{a}^{\prime}\right) \gtrless M_{k+l}^{M S}\left(\mathbf{s}, \mathbf{a}^{\prime \prime}\right)
$$

produces the same decision as (8). Hence iteration of (12) will produce the same decision as Ungerboeck's algorithm, that is MLSE. QED

The theorem can be proved by simpler manipulations by showing that the test (12) produces the same decision as the test of the WMF-MLSE. However, the matched filter always exists, while the WMF does not exist when $S(\omega)$ is null over some measurable interval. Hence the present proof, which is based on the matched filter, demonstrates that the MSWMF leads to MLSE also when the WMF does not exist.

\section{REFERENCES}

[1] A. Duel-Hallen and C. Heegard, "Delayed decisionfeedback sequence estimation," IEEE Trans. Commun., vol. COM-37, pp. 428-436, May 1989.

[2] G. D. Forney, "Maximum-likelihood sequence estimation of digital sequences in the presence of intersymbol interference," IEEE Trans. Inform. Theory, vol. IT-18, pp. 363-378, May 1972.

[3] G. Ungerboeck, "Adaptive maximum-likelihood receiver for carrier modulated data-transmission systems," IEEE Trans. Commun., vol. COM-22, pp. 624-636, May 1974.

[4] L. C. Barbosa, "Maximum likelihood sequence estimators: a geometric view," IEEE Trans. Inform. Theory, vol. IT-35, pp. 419-427, Mar. 1989.

[5] J. Salz, "Optimum mean-square decision feedback equalization," Bell Syst. Tech. J., vol. 52, no. 8, pp. 1341-1373, Dec. 1973.

[6] R. Raheli, A. Polydoros, and C.-K. Tzou, "Persurvivor processing: a general approach to MLSE in uncertain environments," IEEE Trans. Commun., vol. COM-43, pp. 354-364, Feb./Mar./Apr. 1995.

[7] J. M. Cioffi, G. P. Dudevoir, M. V. Eyuboglu, and G. D. Forney, "MMSE decision-feedback equalizers and coding-part I: equalization results," IEEE Trans. Commun., vol. COM-43, pp. 2582-2594, Oct. 1995.

[8] R. R. Anderson and G. J. Foschini, "The minimum distance for MLSE digital data systems of limited complexity," IEEE Trans. Inform. Theory, vol. IT21, pp. 544-551, Sept. 1975. 